\title{
The Relationship between Nature of Social Change, Age, and Position of New Neurons and Their Survival in Adult Zebra Finch Brain
}

\author{
Einat Adar, ${ }^{1}$ Fernando Nottebohm, ${ }^{2}$ and Anat Barnea ${ }^{3}$ \\ ${ }^{1}$ Department of Zoology, Tel-Aviv University, Tel-Aviv 61391, Israel, ${ }^{2}$ The Rockefeller University Field Research Center, Millbrook, New York 12545 , and \\ ${ }^{3}$ Department of Natural and Life Sciences, The Open University of Israel, Raanana 43107, Israel
}

\begin{abstract}
Some kinds of neurons are spontaneously recruited in the intact, healthy adult brain, but the variables that affect their survival are not always clear. We show that in caudal nidopallium of adult male zebra finches, the rostrocaudal position of newly recruited neurons, their age (1 vs 3 months), and the nature of social change (complex vs simple) after the neurons were born affect their survival. Greater social complexity promoted the survival of younger new neurons, and the demise of older ones; a less marked social change promoted the survival of older new neurons. These effects were position dependent. We suggest that functional correlations between new neuron recruitment/survival and its inferred benefit to the animal might be better perceived when taking into account the position of cells, their age at the time of life style changes, and the nature and magnitude of the life style change.
\end{abstract}

Key words: songbirds; neuronal age; neuronal replacement; adult brain; nidopallium caudale; information load

\section{Introduction}

New neurons are recruited into the forebrains of adult birds (Nottebohm, 1985; Alvarez-Buylla et al., 1994; Ling et al., 1997) and mammals (Altman, 1962, 1963, 1967, 1969; Lois and Alvarez-Buylla, 1994; Eriksson et al., 1998; Gould and Gross, 2002; Rakic, 2002; Sanai et al., 2004; Dayer et al., 2005). The origin and birth site of these neurons, the identity of their stem cells, their migratory phenotype, and details about their migration have been partially described in each of these two broad groups (Alvarez-Buylla and Nottebohm, 1988; Alvarez-Buylla et al., 1998; Doetsch et al., 1999; Seri et al., 2001; Alvarez-Buylla and Garcia-Verdugo, 2002; Merkle et al., 2004). The new neurons connect to existing circuits and become part of the working brain (Paton and Nottebohm, 1984; van Praag et al., 2002). However, this fate is not guaranteed, for in some parts of the brain, at least, most of the new cells are discarded before reaching destination or soon thereafter (Alvarez-Buylla and Nottebohm, 1988). Extent of use determines their survival, an effect that is mediated by brainderived neurotrophic factor (BDNF) upregulation by pathway use (Rasika et al., 1999; Li et al., 2000; Alvarez-Borda et al., 2004). In the songbird HVC, new cells are recruited with no net gain in neuron numbers (Kirn and Nottebohm, 1993; Kirn et al., 1994; Scharff et al., 2000), but in other systems such as the adult mam-

Received Dec. 23, 2007; revised April 13, 2008; accepted April 16, 2008

This work was supported by Israel Science Foundation Grant 481/04 and the Open University Research Fund (A.B.). We thank Arnon Lotem for very useful discussions and his help in various aspects of the work. Thanks are due also to the staff of the Meyer Segals Garden for Zoological Research at Tel-Aviv University for their technical help, and to I. Gelernter for her help with statistical analysis. F. Nottebohm was supported by Rockefeller University.

Correspondence should be addressed to Anat Barnea, Department of Natural and Life Sciences, P.0. Box 808, 108 Ravutski Street, The Open University of Israel, Raanana 43107, Israel. E-mail: anatba@openu.ac.il.

DOI:10.1523/JNEUROSCI.5706-07.2008

Copyright $\odot 2008$ Society for Neuroscience $\quad$ 0270-6474/08/285394-07\$15.00/0 malian olfactory bulb, there is a net gain in neuron numbers (Ninkovic et al., 2007). We still know little about the conditions that influence new neuron survival and how this relates to the life of free-ranging animals. The functional significance of new neurons in adult brains has been questioned (Leuner et al., 2006).

Our previous studies looked at new neuron counts in the forebrain of an adult songbird, the zebra finch (Taeniopygia guttata): Lipkind et al. (2002) showed that social environment affected these counts in the nidopallium caudale (NC), HVC, and area X. Birds killed $40 \mathrm{~d}$ after treatment with a cell birth marker had more new neurons if soon after this treatment they were placed in a large social group than if they were housed singly or in pairs. Barnea et al. (2006) repeated this protocol, focusing on the NC and the hippocampal complex (HC), and counted new neurons 40, 60, and $150 \mathrm{~d}$ after social change. Again, new neuron counts were higher in birds housed communally than in birds housed singly and were higher at earlier than at later survival times. The new neurons lived longer in rostral than in caudal NC and their turnover was more marked in NC than in HC. The present report explores how the survival of new neurons of different ages, in different locations, is affected by a simple or complex social change. We use our results to speculate on the significance of neuronal turnover.

\section{Materials and Methods}

Experimental design. Male zebra finches were reared in three outdoor breeding colonies at the Meier Segals Garden for Zoological Research at Tel-Aviv University and banded as fledglings with one numbered plastic ring for individual identification. At $45-60 \mathrm{~d}$ of age, when they became independent and could also be sexed by their plumage, each experimental male was removed from its native colony and housed outdoors in a standard cage $(65 \times 35 \times 45 \mathrm{~cm})$ with three other unrelated individuals. This was done to avoid stress that, in this very social species, might result 
from isolation. The three strangers consisted of another juvenile male of the same age, and two adults (a male and a female $>150 \mathrm{~d}$ old). Birds in these cages could only hear or see the other zebra finches in the same cage.

Experimental males stayed in that setting until they were $\sim 120 \mathrm{~d}$ old. At that time, each one of them got five daily intramuscular injections of $130 \mu \mathrm{l}$ of 5-bromo-2'-deoxyuridine (BrdU; SigmaUltra, diluted 10 $\mathrm{mg} / \mathrm{ml}$ in sterile water; Sigma) as a cellular birth-date maker. The BrdU doses that we used were similar to those used by others studying adult neurogenesis in birds (Wang et al., 2002; Wilbrecht et al., 2002; Pytte et al., 2007). After the BrdU injections, the birds were kept under the above conditions for 30 or 90 additional days. Then, each male was placed in a large outdoor aviary $(1.5 \times 1.5 \times 2 \mathrm{~m})$ where it encountered either of two social environments: simple (with one unfamiliar adult female), or complex (with a pre-existing group of $40-45$ adults of both sexes, all of them strangers to the experimental bird). We shall refer to these two social settings as "simple" and "complex." The walls of these aviaries were covered with burlap and the aviary had a roof of opaque plastic. The distance between aviaries was approximately $50 \mathrm{~m}$. Because of all these conditions birds in any given aviary could not hear nor see those in other aviaries. The interior arrangement (perches, food, and water dishes) was the same for all aviaries. Experimental birds stayed in the new social environments for $40 \mathrm{~d}$.

All cages and aviaries were exposed to natural illumination conditions that changed seasonally (10.1-14.7 h of light per day). The range of mean daily temperature was $12-30^{\circ} \mathrm{C}$. Our breeding stock is able to breed any time of year under these conditions. For this reason and because individuals for each of the experimental groups were obtained at all times of the year, seasonal changes in temperature and photoperiod were unlikely to have affected the outcome of our study.

Recapitulating, the groups and their names were as follows. In the group C1M (complex, 1 month; $n=7$ ), there was a 1 month interval between the last BrdU injection and the exposure of the experimental male to a new social setting where it encountered $40-45$ adult males and females already in residence. In the group S1M (simple, 1 month; $n=6$ ), treatment was as above, except that the experimental male was exposed to a new social setting where it encountered a single female it had not seen before. The group C3M (complex, 3 months; $n=7$ ) was the same as $\mathrm{C} 1 \mathrm{M}$, but exposure to a new social setting occurred 3 months after the BrdU injections. The group S3M (simple, 3 months; $n=6$ ), was the same as $\mathrm{S} 1 \mathrm{M}$, but the male was placed with a novel female companion 3 months after the BrdU treatment.

The times and ages that matter for interpreting the results we present are as follows. BrdU in all groups was given at approximately posthatching day 120. Exposure to a new social setting occurred either 1 month (age $150 \mathrm{~d}$ ) or 3 months (age $210 \mathrm{~d}$ ) after end of BrdU treatment. Birds in all cases were killed $40 \mathrm{~d}$ after being introduced to the new social setting, either at $190 \mathrm{~d}$ or at $250 \mathrm{~d}$ of age. Previous work with another songbird, canaries (Serinus canaria), showed that, depending on the part of the forebrain studied, neurons born in adult telencephalon can take from $8 \mathrm{~d}$ (Kirn et al., 1999) to 30-40 d (Alvarez-Buylla and Nottebohm, 1988) to reach their final destination, there to acquire their postmigratory phenotype. Thus, in C1M and S1M, exposure to a new social setting probably occurred soon after the new $\mathrm{BrdU}^{+}$neurons had arrived at their destinations, while they were still establishing their connections and even while stragglers were still arriving. The introduction to a new social setting in C3M and S3M birds probably occurred well after the new neurons had ended their migration and acquired their adult connections and phenotype.

Histology and immunohistochemistry. Forty days after the exposure to the new social setting, experimental birds were weighed, then killed with an overdose of anesthesia $(0.06 \mathrm{ml}$ of ketalar diluted 10 times followed by $0.06 \mathrm{ml}$ of xylazine) and fixed with an intracardiac perfusion with saline $(\mathrm{NaCl} 0.9 \%)$ followed by $4 \%$ paraformaldehyde in $0.1 \mathrm{M}$ phosphate buffer (PB; pH 7.4). Then, brains were removed, weighed, placed in the same fixative for $1 \mathrm{~h}$, and transferred into $\mathrm{PB}$ in $4^{\circ} \mathrm{C}$. After $1-4 \mathrm{~d}$, brains were dehydrated in alcohols, embedded in polyethylene glycol, blocked, and cut transversely at $6 \mu \mathrm{m}$ intervals. Serial sections (every 20; i.e., at intervals of $120 \mu \mathrm{m}$ ) were mounted on slides (Superfrost/PLUS), using a solution of $0.1 \%$ BSA (albumin bovine, minimum 98\%) in PBS.
Sections were incubated for $10 \mathrm{~min}$ in $0.01 \mathrm{~m}$ citrate buffer ( $\mathrm{pH} 5.6-6$ ) at $90-95^{\circ} \mathrm{C}$ to denature the DNA (which makes the BrdU more accessible to the antibody), washed in PB for $5 \mathrm{~min}$, and incubated for $2 \mathrm{~min}$ in weak pepsin (pepsin stock; Sigma, 2.5\% in PBS $1 \mathrm{M}$ ) diluted $1: 19$ in $0.1 \mathrm{~N}$ $\mathrm{HCl}$ at room temperature (RT). After washing (PB, three times, $5 \mathrm{~min}$ each), sections were incubated in $3 \% \mathrm{H}_{2} \mathrm{O}_{2}$ in $\mathrm{PB}$ for 20 min and then washed with $\mathrm{PB}$ twice, 5 min each. Blocking of unspecific binding sites was done by incubating for $1 \mathrm{~h}$ in blocking buffer (PB containing 10\% normal horse serum and $0.3 \%$ Triton X-100). Then, sections were incubated overnight at $4^{\circ} \mathrm{C}$, with the primary antibody, rat anti-BrdU IgG2a (Serotec; diluted 1:200). The next day, the sections were washed and incubated for $2 \mathrm{~h}$ at RT with the secondary antibody $\mathrm{F}(\mathrm{ab})_{2}$ donkey anti-rat IgG-Cy3 (Jackson ImmunoResearch; diluted 1:200), which stains $\mathrm{BrdU}^{+}$cells with fluorescent red. Sections were washed again and incubated in blocking buffer for $30 \mathrm{~min}$. Then, the sections were incubated overnight at $4^{\circ} \mathrm{C}$ with another primary antibody that affixes specifically to neurons: anti-HuC/HuD mouse $\operatorname{IgG} 2_{b}$, monoclonal $16 \mathrm{~A} 11$ (Invitrogen; diluted 1:200). The next day, sections were washed and incubated for $2 \mathrm{~h}$ at RT with the secondary antibody biotinylated antimouse IgG $(\mathrm{H}+\mathrm{L})$ made in horse (Vector Laboratories; diluted 1:200). Sections were then washed and treated for $30 \mathrm{~min}$ with avidin biotin peroxidase complex (ABC R.T.U. Vectastain kit; Vector Laboratories) and incubated for 5-7 min with 16\% DAB (3,3' -diaminobenzidine tetrahydrochloride; Sigma) in $\mathrm{PB}$, which, in the presence of $0.01 \% \mathrm{H}_{2} \mathrm{O}_{2}$, stains the neurons in the tissue brown. Then, sections were washed with $\mathrm{dH}_{2} \mathrm{O}$, followed by a PB wash and coverslipped with Aqua-Poly/Mount glue (Polysciences). Slides were kept in the dark at $4^{\circ} \mathrm{C}$ until mapping.

Mapping and quantification. The immunohistochemistry procedure described above yielded neurons that were stained brown, and $\mathrm{BrdU}^{+}$ nuclei that were stained with fluorescent red. A similar procedure has been applied previously by others (Wilbrecht et al., 2002). Our counts of new neurons counted cells with brown cytoplasm and a red fluorescent nucleus (Fig. 1). We analyzed several dozens of such neurons by a confocal microscope to ensure colocalization of both markers. This was done by finding substantial evidence that the red fluorescent stainings had the typical shape, size, and appearance of nuclei, and that they were surrounded (at least by half or more) by brown staining.

We made our counts of new neurons in three brain regions: the NC, HVC, and HC. The NC was chosen because it includes auditory relays that probably play a role in vocal communication and in the processing of other types of auditory inputs (Vates et al., 1996; Mello et al., 1998). The lateral part of this brain region is also activated by visual stimulation and heightened arousal (such as when a male is chased about in its living quarters) and by courtship behavior (when it is presented for the first time, after weeks of isolation, with a sexual partner). Such conditions result in lateral nidopallium in a rise in c-fos expression and irreversible increase in spine numbers in neurons (Bischof and Rollenhagen, 1999; Sadananda and Bischof, 2002, 2006). HVC is part of the song control system and is also involved in vocal communication (for review, see Nottebohm, 2002). The HC is known to be involved in the processing of spatial information (Krebs et al., 1989), and there is no evidence that auditory input reaches it. However, in a previous study (Barnea et al., 2006), we found that new neuronal recruitment and survival in this brain region was affected by the social setting in which birds had been placed. All of these three brain regions were investigated in our previous studies and new neuron counts in them found to be affected by social manipulation. The anatomical relation between these parts is shown in Figure 2. All of these three brain regions have boundaries that are easy to recognize in transverse sections: for the NC and HVC, we followed the criteria described by Lipkind et al. (2002), and for the HC those described by Barnea et al. (2006).

NC stretches rostrocaudally over a distance of $1200 \mu \mathrm{m}$. From this area, five sections were sampled for our counts of $\mathrm{BrdU}^{+}$neurons. The middle section went through the robust nucleus of arcopallium at its largest diameter; in the canary atlas (Stokes et al., 1974), this would correspond to level P1.2. Then, two more sections were obtained, rostrally and caudally, respectively, at distances of 360 and $600 \mu \mathrm{m}$ from the middle section. In HVC, which is smaller $(240-720 \mu \mathrm{m}$ along its rostrocaudal axis), counts of $\mathrm{BrdU}^{+}$neurons were made in all sections in which 
HVC appeared (three to seven per brain). Rostrally, the first section would correspond to AP0.0 in the canary atlas (Stokes et al., 1974). In $\mathrm{HC}$, which is narrow and stretches over 2000 $\mu \mathrm{m}$ in the rostrocaudal axis, five sections were sampled per brain. Rostrally, the first section was the one in which HC first appears (corresponds to A4.0 in the canary atlas) (Stokes et al., 1974), and the fifth and most caudal was the one that corresponds to A0.2 in the canary atlas. The other three in-between sections were evenly distributed so that, on average, the distance between each mapped section and the next one was $480 \mu \mathrm{m}$.

We used a computerized brain-mapping system (Neurolucida; Stereo Investigator; MicroBrightField) to draw boundaries of the NC, $\mathrm{HVC}$, and $\mathrm{HC}$ in each section sampled, enter the position of each labeled neuron, count neurons and quantify other neuronal parameters, as described below. All mapping was done "blind" as to the experimental conditions. Preliminary mapping in all regions showed no hemispheric differences in the number of labeled neurons per cubic millimeter. Therefore, for characterizing neuronal survival in each of the experimental groups we mapped sections only from the left hemisphere.

Each section sampled in the NC, HVC, and HC was completely scanned, using the Neurolucida meander scan probe. All $\mathrm{BrdU}^{+}$neurons were mapped and counted in the sections sampled, using a $63 \times$ objective. We also measured nuclear diameter of all of these neurons. From the knowledge of section thickness and mean nuclear diameter of labeled neurons in a certain brain region, using the Abercrombie stereological correction equation (Guillery and Herrup, 1997), we could estimate the number of $\mathrm{BrdU}^{+}$neurons per cubic millimeter in each of the three brain regions. In NC, the estimated number was obtained for each section separately. In the HC and HVC, data from all sections mapped for each brain were pooled so that each brain was represented by a single estimate of the number of $\mathrm{BrdU}^{+}$neurons per cubic millimeter. This was done because labeling in HVC and HC sections was low, and some sections did not have any labeled neurons.

To meaningfully compare the number of $\mathrm{BrdU}^{+}$neurons per cubic millimeter in a given brain region between experimental groups, we needed to assume that the total numbers of neurons (labeled and unlabeled) per cubic millimeter in that region did not vary between experimental groups. However, this had already been tested and proven to be the case in three previous studies (Lipkind et al., 2002; Barnea et al., 2006; Barkan et al., 2007) for all three brain regions. Moreover, a previous study from our laboratory further confirmed this outcome for $\mathrm{NC}$, this time by using tissue which was treated with the neuronal marker $\mathrm{Hu}$, just as was done with our present material (M. Pnini, L. Lotem, F. Nottebohm, and A. Barnea, unpublished data).

We did not sample systematically for possible mediolateral differences in the occurrence of new neurons in the three structures studied. However, in a previous publication (Barnea et al., 2006), when we generally looked at medial and lateral domains in the NC, we did not detect any particular clustering around any anatomical landmarks.

Statistical analysis. All data that were expressed as number of neurons per cubic millimeter were transformed before the statistical analysis by using the square root transformation. [These kinds of data, number of discrete elements per unit, tend to have a poison distribution, and the suitable transformation for such a case is the square root transformation (Sokal and Rholf, 1995).] For the NC, we tested for significance of group differences in numbers of $\mathrm{BrdU}^{+}$neurons using a two-way ANOVA with repeated measures, in which the variables were simple or complex social setting, 1 or 3 months age of $\mathrm{BrdU}^{+}$neurons when exposed to the new environment, and section number as the repeated measure. For the HC and HVC, we used a two-way ANOVA, using as variables simple or complex social setting, and 1 or 3 months age of $\mathrm{BrdU}^{+}$neurons when exposed to the new environment.

The smaller areas of the HC and HVC than those of the NC resulted, as explained above, in some HC and HVC sections having no labeled neurons, which then limited our ability to test for rostrocaudal gradients in numbers of $\mathrm{BrdU}^{+}$neurons, and this made it harder to compare our $\mathrm{HC}$, $\mathrm{HVC}$, and NC results using the same formula for all three. To overcome this limitation, we also analyzed the $\mathrm{NC}$ data pooling the results from all five sections.

\section{Results}

\section{Nidopallium caudale}

Figure 3 shows, in rostrocaudal order, the number of $\mathrm{BrdU}^{+}$ neurons per cubic millimeter in each of five NC sections sampled. Our estimates of number of $\mathrm{BrdU}^{+}$neurons per cubic millimeter show that there was a significant interaction between (1) the nature of social change and time after BrdU treatment when this social change occurred $\left(F_{(1,22)}=6.89 ; p=0.015\right)$, and (2) the nature of social change and section number $\left(F_{(4,88)}=3.22 ; p=\right.$ $0.016)$; 3$)$ time after BrdU treatment when social change occurred and section number $\left(F_{(4,88)}=3.08 ; p=0.02\right)$. From this, we infer that all three variables (nature of social change, time after BrdU treatment when social change occurred, and position along the NC rostrocaudal axis) had a significant effect on estimates of $\mathrm{BrdU}^{+}$neurons per cubic millimeter. Because of these three significant interactions we could not pool data from all sections or perform meaningful post hoc comparisons to test for differences between experimental groups. Instead, we interpret below the way in which each interaction affected new neuronal survival.

What, then, is the nature of this effect? We assume that for each of the section levels (1-5), the number of $\mathrm{BrdU}^{+}$neurons per cubic millimeter was initially the same for all experimental groups because this labeling took place before the birds were assigned to the different groups. Therefore, we assume that the 

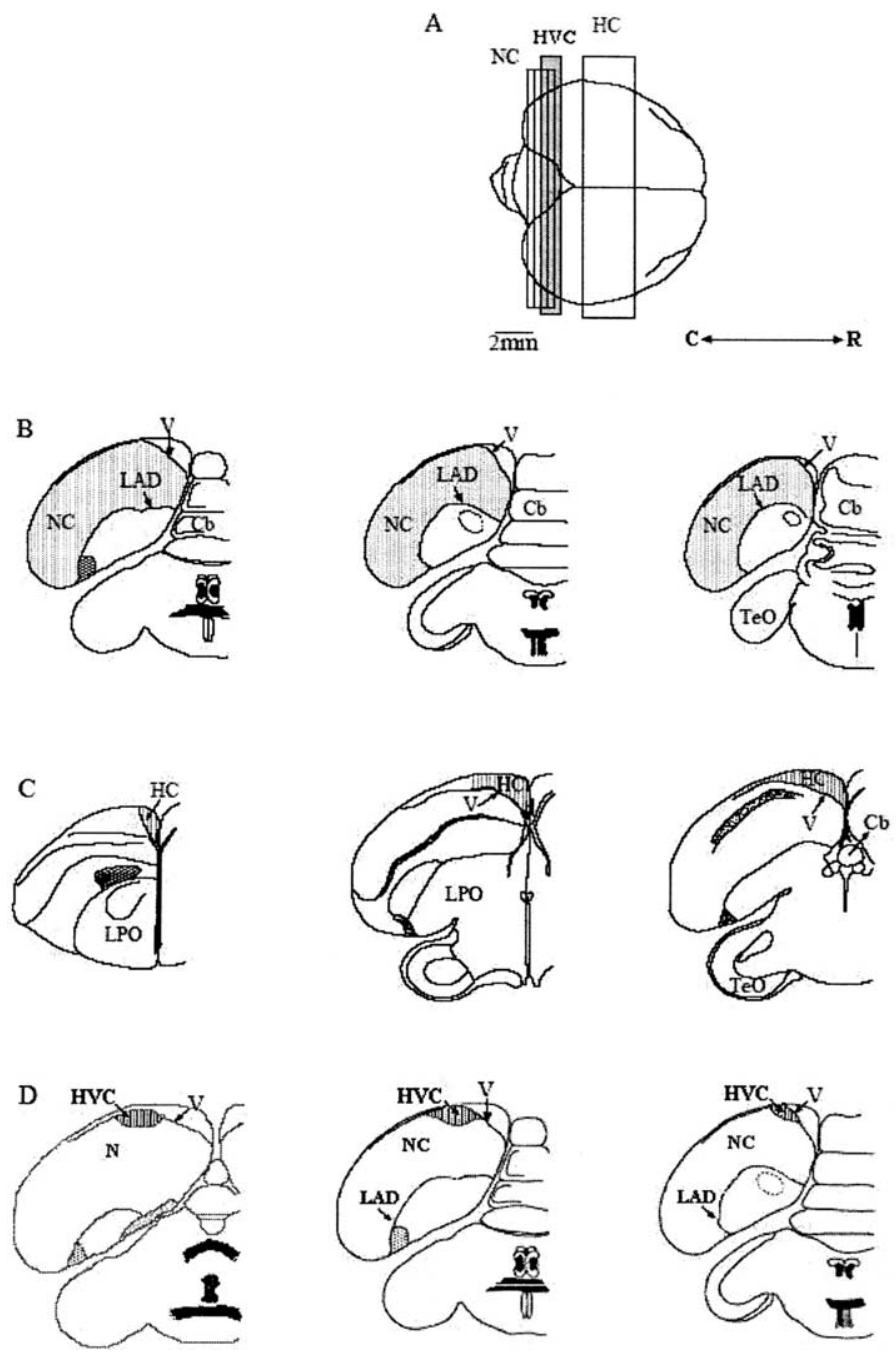

Figure 2. A, Top schematic view of the brain of an adult zebra finch male; caudal is to the left, rostral is to the right. We indicate the range within which frontal sections were taken from the NC, HVC, and HC. $\boldsymbol{B}-\boldsymbol{D}$, A few sections were sampled in each brain region along the rostrocaudal axis (for details, see Materials and Methods); however, here only three are shown: the most rostral, the middle, and the most caudal ones (from left to right) in NC (B), HC ( $\boldsymbol{C}$, and HVC (D). Cb, Cerebellum; LAD, lamina arcopallialis dorsalis; Te0, tectum opticum; V, lateral ventricle; LPO, lobus parolfactorius [now referred to as medial striatum; nomenclature is according to Reiner et al. (2004)].

highest labeling seen for a section level in any of the experimental groups was also the one closest to the original labeling. Because this highest level is very similar in all five sections (range, 190230 labeled neurons $/ \mathrm{mm}^{3}$ ) (Fig. 3), we infer that initial recruitment was similar in all five sections and that the differences seen among experimental groups resulted from survival differences after social change occurred. It is unlikely that all $\mathrm{BrdU}^{+}$neurons survived in any section level of any of the experimental groups, so that we are just talking about relative differences in the fraction that survived. Thus, to answer the question at the beginning of this paragraph, we will interpret the above interactions by looking at the data from Figure 3, each time from a different perspective:

\section{First interaction}

As indicated above, analysis of the data in Figure 3 showed that the number of $\mathrm{BrdU}^{+}$neurons was significantly affected by the interaction between nature of social change and the age of $\mathrm{BrdU}^{+}$ neurons at the time social change occurred. Figure 4, which is derived from Figure 3, better demonstrates this interaction by

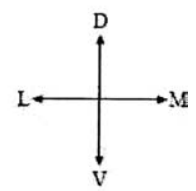

presenting only theses two variables. The outcome of this interaction is that a new neuron in $\mathrm{NC}$ was more likely to survive if it was young ( 1 month) and the new social setting was complex, or if it was old (3 months) and the new social setting was simple.

\section{Second interaction}

The number of $\mathrm{BrdU}^{+}$neurons was affected by the interaction between section number and the nature of social change, as shown by the data in Figure 3. Figure 5, which is derived from Figure 3, better demonstrates this interaction by presenting rostrocaudal numbers of $\mathrm{BrdU}^{+}$neurons per cubic millimeter in each experimental group. The greatest rostrocaudal differences in the survival of the new neurons occurred in the birds placed in the complex social setting 1 month after BrdU treatment (group C1M). Apparently, this social change had a greater effect on the mortality of new neurons at the rostral than at the caudal end of the NC, resulting in a rostrocaudal survival gradient. Rostrocaudal differences in the other three groups were less obvious. Summing up this interaction, survival of 1-month-old NC neurons was favored by social complexity and the more so the more caudal the section.

\section{Third interaction}

The third interaction was between age of the young neurons (equal to time after BrdU treatment) and section number. Put differently, the rostrocaudal position of the sections sampled determined, at different neuronal ages, the number of BrdU neurons counted. This relation applied particularly to birds in the group whose social environment became more complex 1 month after BrdU treatment (C1M), as discussed for the second interaction and demonstrated in Figure 5. In those birds, the complex environment determined a twofold reduction in the number of $\mathrm{BrdU}^{+}$neurons rostrally, an effect that was absent when exposure to a more complex social environment occurred 3 months after BrdU treatment (C3M).

When we pooled the data from all five NC sections, to make the results more comparable with those of HVC and HC (see Materials and Methods), the first interaction, between nature of social change and neuronal age, remained significant $\left(F_{(1,24)}=\right.$ 7.37; $p=0.012)$.

\section{HVC and HC}

No significant differences were found in the number of $\mathrm{BrdU}^{+}$ neurons per cubic millimeter in HVC and HC between experimental groups $\left(F_{(1,24)}=0.37, p=0.55\right.$ and $F_{(1,24)}=1.48, p=$ 0.24 , respectively, for complex vs simple social setting; $F_{(1,24)}=$ $0.003, p=0.96$ and $F_{(1,24)}=0.52, p=0.48$, respectively, for 1 month vs 3 months age of $\mathrm{BrdU}^{+}$neurons when exposed to a new social setting), and no interaction was found between nature of 

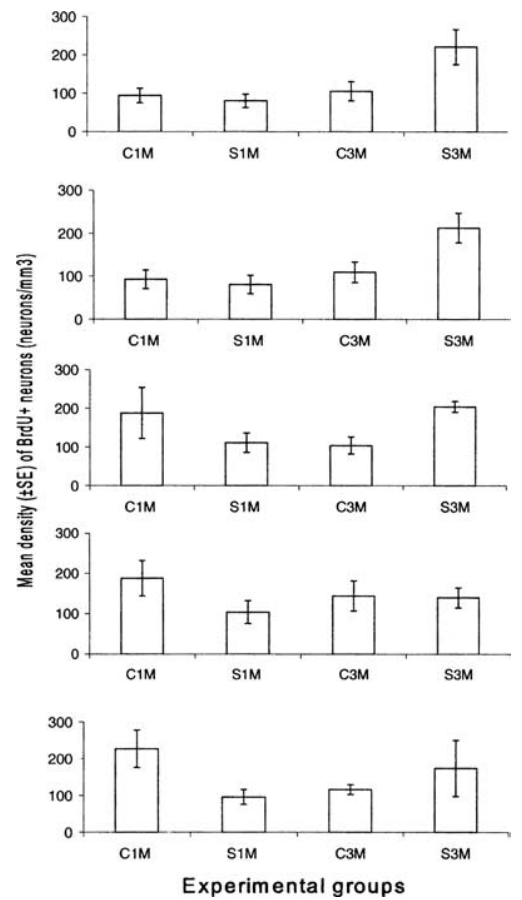

Figure 3. Mean density (neurons per cubic millimeter $\pm \mathrm{SE}$ ) of $\mathrm{BrdU}^{+}$neurons in $\mathrm{NC}$, in five sections (from top to bottom in rostrocaudal order) in brains of males that were exposed to different social environments: (1M, S1M, C3M, S3M (for details, see Results). The sample size in each group was, respectively, 7, 6, 7, and 6 .

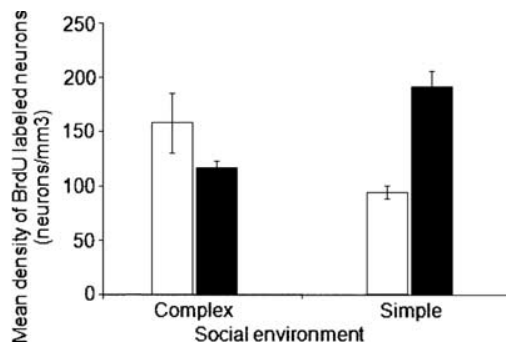

Figure 4. Mean density (neurons per cubic millimeter $\pm \mathrm{SE}$ ) of $\mathrm{BrdU}^{+}$neurons in the $\mathrm{NC}$, in brains of males that were exposed to a complex or simple social environment. White bars, Groups that were exposed to the new social setting 1 month after BrdU treatment (C1M and S1M); black bars, groups that were exposed to the new social setting 3 months after BrdU treatment (C3M and S3M). This figure is derived from Figure 3 to show the interaction between social setting and neuronal age (for details, see Results). The sample size in each group was, respectively, $7,6,7$, and 6 .

social change and neuronal age $\left(F_{(1,24)}=2.46, p=0.13\right.$ and $F_{(1,24)}$ $=1.03, p=0.32$, respectively).

\section{Discussion}

We compared new neuron survival when birds that had been held in a stable social setting with three other individuals were moved to a simple (just a female partner) or complex (45 other zebra finches) social setting. We did not compare an absence of social change with exposure to a new social setting, but rather the effect that each of the two new social settings had on survival, $40 \mathrm{~d}$ after social change, of new neurons that, at the time of social change, were 1 or 3 months old. Thus, the results presented relate to how nature of social change and its timing (neuronal age) affect short term (40 d) new neuronal survival.

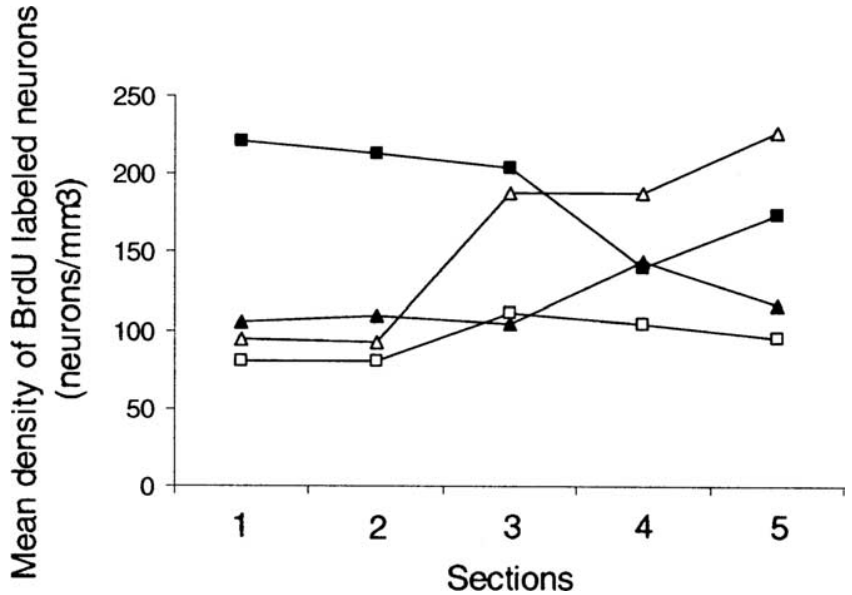

Figure 5. Mean density (neurons per cubic millimeter) of $\mathrm{BrdU}^{+}$neurons in five sections in the NC, in brains of males that were exposed to different social environments: $\triangle, C 1 M ; \square, S 1 M$; $\boldsymbol{\Delta}, \mathrm{C} 3 \mathrm{M} ; \mathbf{\square}, \mathrm{S3}$ M. This figure is derived from Figure 3 to demonstrate the interaction between position along the NC rostrocaudal axis (section number) and social setting (for details, see Results). Lines are drawn for descriptive purposes only. The sample size in each group was, respectively, 7,6, 7, and 6 .

\section{Relation between neuronal age and neuronal survival}

Our previous studies (Lipkind et al., 2002; Barnea et al., 2006) looked at the effect that exposure of birds to a new social environment had on recruitment and survival of neurons born immediately before that change. In those experiments, the new neurons had probably just started migrating to their final destination in the brain. In contrast, the present study looks at the effect that exposure to a new social setting has on survival of neurons born 1 or 3 months earlier. In the present report the new neurons were either at the end of migration and starting to get connected (1 month), or had been connected to existing circuits for several weeks. Our results show that the 1-month-old NC neurons were more likely to survive if the bird was exposed to a complex social setting than if it was exposed to a simple one, and the reverse was true if the neurons were 3 months old (Fig. 4). The explanation for this outcome could be that young new neurons which are not yet committed to a specific job are more readily selected for a new job. The young new neurons may be able to better process and store new information and, as a result, they may be used more than older neurons. Increased use of replaceable neurons may then promote their survival (Li et al., 2000). We hypothesize that the greater the amount of new information, the greater the proportion of young new neurons that may, in this manner, find employment and subsequent survival. However, when dealing with older new neurons, a surge in new information may be more likely to result in their disuse and, therefore, demise, because these neurons are more "set in their ways," committed to specific roles that are incompatible with the new demands. The resulting vacancies will then promote the recruitment of more new neurons of the same kind (Scharff et al., 2000) that, as we have suggested above, may then be better able to cope with the new situation. If this occurs in a part of the adult brain for which the total number of neurons is set (and we do not have this information for the NC) then, as described for HVC (Kirn and Nottebohm, 1993; Kirn et al., 1994; Scharff et al., 2000), there is an ongoing process of neuronal replacement whereby new information promotes the death of older neurons and the recruitment and survival of young ones. That, we believe, is the central insight suggested by the present study.

In a colonial species such as the zebra finch, living just with a 
single new companion (simple social environment) may place modest demands on the capacity of the brain to process social information, a situation that therefore may not require the elimination of many pre-existing neurons (S3M) (Figs. 4, 5). This same modest demand for processing new social information may have resulted in the underuse of a majority of 1-month-old NC neurons in birds housed with a single partner, resulting in the reduced survival of these cells (S1M) (Figs. 4, 5). There is a precedent for the relation between reduced use and increased mortality in neurons (Li et al., 2000). Moreover, the differential survival of 1-month-old NC neurons in birds presented with a complex or simple social setting, as described above (C1M vs S1M) (Fig. 4), is congruent with results from our previous report (Lipkind et al., 2002) in which the new neurons were even younger (just a few days old) at the time of social change.

We found no significant group differences in numbers of $\mathrm{HVC}$ and $\mathrm{HC} \mathrm{BrdU}{ }^{+}$neurons, unlike the observations of Lipkind et al. (2002) and Barnea et al. (2006), which showed a correlation between an increase in social complexity and increased numbers of new neurons in various brain regions of adult zebra finches, including HVC and HC. A later study (Adar et al., 2008) suggested that the increased recruitment of new HVC neurons resulted from an increase in the amount and diversity of auditory input, because males placed in a more complex social setting sang less than those housed with just a female. However, in those previous studies, the new neurons were younger when the bird was placed in the new social setting: the birth-date marker had been given immediately before that event, and this could be the reason for the different results between the present study and the earlier ones.

\section{Relation between anatomical location in the brain and neuronal survival}

It is of interest that the effect of social complexity on survival of young (1 month old) neurons showed a rostrocaudal gradient: survival of the new neurons in the more caudal sections benefited from the increase in social complexity (Fig. 5). This observation lends itself to three very different interpretations: (1) the more caudal neurons were not needed for analysis of new social information and hence were immune to the increased information load; (2) they were able to respond to the new information with their existing connections; and (3) they were able to modify their connections so as to handle the new load. We believe that the first interpretation is unlikely because the same cells were less likely to survive in the simpler social setting, suggesting that use and disuse may have accounted for the differential survival. Our data do not allow us to discriminate between interpretations 2 and 3 .

In a previous publication (Barnea et al., 2006), we also found differential survival rates of new neurons recruited at different rostrocaudal NC levels; in that work, the new neurons were younger than the ones in the present study. We argued then that these differences reflected an "anatomical representation of time." The idea was that parts of the brain that are more sensitive to novelty of information are likely to act as repositories for more recent events, whereas those more resistant to changes in information are likely to hold older memories. That interpretation remains unproven, but our present results refine this idea: we show that, depending on the age of the neuronal cohort tracked, a change toward greater social complexity can affirm or challenge the survival of new neurons (Fig. 4). In parts of the brain populated by replaceable neurons, previous events may determine how recently cohorts of new neurons were incorporated there and, therefore, how ready they will be to assimilate new informa- tion and survive. If so, then the nature and timing of earlier events may determine where new events will be more strongly represented. It would be of interest to know whether marked successive changes in environmental information would result in a lower mean age of replaceable neurons. Of course, an excess of these switches could lead to little permanent information. Because our experiment was structured to compare the effect that exposing adult birds to a novel complex or simple social setting would have on the survival of 1-month- and 3-month-old neurons, the quiltwork of our results is incomplete. Future work should sample more widely the effect that environmental/behavioral change has on recruitment of neurons of different ages, in different locations within a same brain region, and the duration of survivorship conferred on the new neurons.

\section{Overview}

We conclude that questions about neuronal turnover may, in other instances as well as in the present, have to be informed about several variables, including the exact region that is tested, the age of the neurons tested, and the nature of the environmental change. If the study samples a large area and averages the results from all cell ages and locations sampled, real effects might go unnoticed, thus rendering the strategy the brain uses to replace its neurons totally incomprehensible. This risk increases when sample sizes are modest, so that only the most striking effects reach statistical significance. It is likely that brains use exquisite calculations in their decision of which cells to replace and which to keep, and under what circumstances and for how long. A cell vested with information may be a humbug or a precious resource. Our results help lay out a hypothetical choreography and rationale for neuronal replacement. Although older replaceable cells, set in their ways, must be eliminated as the animal grapples with a surge of new information, younger new neurons respond to the same surge as a positive stimulus for their survival and, thus, as in societies, one generation replaces the next. Neuronal turnover remains the brain's most radical way of responding to acute changes in the amount and novelty of the information it must process and, perhaps, remember.

\section{References}

Adar E, Lotem A, Barnea A (2008) The effect of social environment on singing behavior in the zebra finch (Taeniopygia guttata) and its implication for neuronal recruitment. Behav Brain Res 187:178-184.

Altman J (1962) Are new neurons formed in the brains of adult mammals? Science 135:1127-1128.

Altman J (1963) Autoradiographic investigation of cell proliferation in the brains of rats and cats. Anat Rec 145:573-591.

Altman J (1967) Postnatal growth and differentiation of the mammalian brain, with implications for a morphological theory of memory. In: The neurosciences. First study program (Quarton GC, Melnechuk T, Schmitt FO, eds), pp 723-743. New York: Rockefeller UP.

Altman J (1969) DNA metabolism and cell proliferation. In: Structural neurochemistry, handbook of neurochemistry, Vol 2 (Lajtha A, ed), pp 137182. New York: Plenum.

Alvarez-Borda B, Haripal B, Nottebohm F (2004) Timing of BDNF exposure affects life expectancy of new neurons. Proc Natl Acad Sci USA 101:3957-3961.

Alvarez-Buylla A, Garcia-Verdugo JM (2002) Neurogenesis in adult subventricular zone. J Neurosci 22:629-634.

Alvarez-Buylla A, Nottebohm F (1988) Migration of young neurons in adult avian brain. Nature 335:353-354.

Alvarez-Buylla A, Ling CY, Yu WS (1994) Contribution of neurons born during embryonic, juvenile and adult life to the brain of adult canaries: regional specificity and delayed birth of neurons in the song control nuclei. J Comp Neurol 347:233-248.

Alvarez-Buylla A, Garcia-Verdugo JM, Mateo AS, Merchant-Larios H (1998) 
Primary neural precursors and intermitotic nuclear migration in the ventricular zone of adult canaries. J Neurosci 18:1020-1037.

Barkan S, Ayali A, Nottebohm F, Barnea A (2007) Neuronal recruitment in adult zebra finch brain during a reproductive cycle. Develop Neurobiol 67:687-701.

Barnea A, Mishal A, Nottebohm F (2006) Social and spatial changes induce multiple survival regimes for new neurons in two regions of the adult brain: an anatomical representation of time? Behav Brain Res 167:63-74.

Bischof, HJ, Rollenhagen A (1999) Behavioural and neurophysiological aspects of sexual imprinting in zebra finches. Behav Brain Res 98:267-276.

Dayer AG, Cleaver KM, Abouantoun T, Cameron HA (2005) New GABAergic interneurons in the adult neocortex and striatum are generated from different precursors. J Cell Biol 168:415-427.

Doetsch F, Caille I, Lim DA, Garcia-Verdugo JM, Alvarez-Buylla A (1999) Subventricular zone astrocytes are neural stem cells in the adult mammalian brain. Cell 97:703-716.

Eriksson PS, Perfilieva E, Bjork-Eriksson T, Alborn AM, Nordborg C, Peterson DA, Gage FH (1998) Neurogenesis in the adult human hippocampus. Nat Med 11:1313-1317.

Gould E, Gross CG (2002) Neurogenesis in adult mammals: some progress and problems. J Neurosci 22:619-623.

Guillery RW, Herrup K (1997) Quantification without pontification. J Comp Neurol 386:2-7.

Kirn JR, Nottebohm F (1993) Direct evidence for loss and replacement of projection neurons in adult canary brain. J Neurosci 13:1654-1663.

Kirn JR, O'Loughlin B, Kasparian S, Nottebohm F (1994) Cell death and neuronal recruitment in the high vocal center of adult male canaries are temporally related to changes in song. Proc Natl Acad Sci USA 19:7844-7848.

Kirn JR, Fishman Y, Sasportas K, Alvarez-Buylla A, Nottebohm F (1999) Fate of new neurons in adult canary high vocal center during the first 30 days after their formation. J Comp Neurol 411:487-494.

Krebs JR, Sherry DF, Healy SD, Perry VH, Vaccarino AL (1989) Hippocampal specialization of food-storing Birds. Proc Natl Acad Sci USA 86:1388-1392.

Leuner B, Gould E, Shors TJ (2006) Is there a link between adult neurogenesis and learning? Hippocampus 16:216-224.

Li XC, Jarvis ED, Alvarez-Borda B, Lim DA, Nottebom F (2000) A relationship between behavior, neurotrophin expression and new neuron survival. Proc Natl Acad Sci USA 97:8584-8589.

Ling CY, Zuo MX, Alvarez-Buylla A, Cheng MF (1997) Neurogenesis in juvenile and adult ring doves. J Comp Neurol 379:300-312.

Lipkind D, Nottebohm F, Rado R, Barnea A (2002) Social change affects the survival of new neurons in the forebrain of adult songbirds. Behav Brain Res 133:31-43.

Lois C, Alvarez-Buylla A (1994) Long-distance neuronal migration in the adult mammalian brain. Science 264:1145-1148.

Mello CV, Vates GE, Okuhata S, Nottebohm F (1998) Descending auditory pathways in the adult male Zebra Finch (Taeniopygia guttata). J Comp Neurol 395:137-160.

Merkle FT, Tramontin AD, Garcia-Verdugo JM, Alvarez-Bylla A (2004) Radial glia give rise to adult neural stem cells in the subventricular zone. Proc Natl Acad Sci USA 101:17528-17532.
Ninkovic J, Mori T, Goetz M (2007) Distinct modes of neuron addition in adult mouse neurogenesis. J Neurosci 27:10906-10911.

Nottebohm F (1985) Neuronal replacement in adulthood. Ann NY Acad Sci 457:143-161.

Nottebohm F (2002) Neuronal replacement in adult brain. Brain Res Bull 57:737-749.

Paton JA, Nottebohm F (1984) Neurons generated in adult brain are recruited into functional circuits. Science 225:1046-1048.

Pytte CL, Gerson M, Miller J, Kirn JR (2007) Increasing stereotopy in adult zebra finch song correlates with a declining rate of adult neurogenesis. Develop Neurobiol 67:1699-1720.

Rakic P (2002) Adult neurogenesis in mammals: an identity crisis. J Neurosci 22:614-618.

Rasika S, Alvarez-Buylla A, Nottebohm F (1999) BDNF mediates the effects of testosterone on the survival of new neurons in an adult brain. Neuron 22:53-62.

Reiner A, Perkel DJ, Bruce LL, Butler AB, Csillag A, Kuenzel W, Medina L, Paxinos G, Shimizu T, Striedter G, Wild M, Ball GF, Durand S, Gunturkun O, Lee DW, Mello CV, Powers A, White SA, Hough G, Kubikova $\mathrm{L}$, et al. (2004) Revised nomenclature for avian telencephalon and some related brainstem nuclei. J Comp Neurol 473:377-414.

Sadananda M, Bischof HJ (2002) Enhanced fos expression in the zebra finch (Taeniopygia guttata) brain following first courtship. J Comp Neurol 448:150-164

Sadananda M, Bischof HJ (2006) C-fos induction in forebrain areas of two different visual pathways during consolidation of sexual imprinting in the zebra finch (Taeniopygia guttata). Behav Brain Res 173:262-267.

Sanai N, Tramontin AD, Quinones-Hinojosa A, Barbaro NM, Gupta N, Kunwar S, Lawton MT, Mcdermott MW, Parsa AT, Garcia-Verdugo JM, Berger MS, Alvarez-Buylla A (2004) Unique astrocyte ribbon in adult human brain contains neural stem cells but lacks chain migration. Nature 427:740-744

Scharff C, Kirn J, Grossman M, Macklis J, Nottebohm F (2000) Targeted neuronal death affects neuronal replacement and vocal behavior in adult songbirds. Neuron 25:481-492.

Seri B, Garcia-Verdugo JM, McEwen BS, Alvarez-Buylla A (2001) Astrocytes give rise to new neurons in the adult mammalian hippocampus. J Neurosci 21:7153-7160.

Sokal RR, Rholf JF (1995) Biometry. New York: Freeman.

Stokes TC, Leonard CM, Nottebohm F (1974) The telencephalon, diencephalon and mesencephalon of the canary, Serinus canaria, in stereotaxic coordinates. J Comp Neurol 156:337-374.

van Praag H, Schinder AF, Christie BR, Toni N, Palmer TD, Gage FH (2002) Functional neurogenesis in the adult hippocampus. Nature 415:1030-1034.

Vates GE, Broome BM, Mello CV, Nottebohm F (1996) Auditory pathways of caudal telencephalon and their relation to the song system of adult male Zebra Finches (Taenopygia guttata). J Comp Neurol 366:613-642.

Wang N, Hurley P, Pytte C, Kirn JR (2002) Vocal control neuron incorporation decreases with age in the adult zebra finch. J Neurosci 22:10864-10870.

Wilbrecht L, Crionas A, Nottebohm F (2002) Experience affects recruitment of new neurons but not adult neuron number. J Neurosci 22:825831. 